$>$ Les maladies auto-immunes comme le lupus érythémateux disséminé sont la conséquence d'une réponse immune contre l'organisme luimême, anormalement considéré comme étranger. Elles se caractérisent par un état inflammatoire et des dommages cellulaires et tissulaires parfois graves. Des défauts de l'autophagie qui touchent les lymphocytes de souris et de patients lupiques ont été mis en évidence. Le peptide synthétique P140/Lupuzor qui cible les voies autophagiques, notamment celles impliquant les protéines chaperonnes, est un candidat-médicament efficace qui ne présente pas d'effet indésirable. II réduit la sur-activation de certaines voies autophagiques et la présentation de peptides antigéniques aux lymphocytes T autoréactifs. <

D'un point de vue étymologique et linguistique, le dictionnaire Larousse nous précise que le terme «système » provient du bas latin systema et du grec sustêma et signifie un «ensemble organisé de principes coordonnés de façon à former un tout scientifique ou un corps de doctrine ». II nous est précisé qu'il s'agit d'un « ensemble d'éléments interagissant entre eux selon certains principes ou règles », qu'un système « est déterminé par la nature de ses éléments constitutifs, les interactions entre ces derniers, sa frontière, ses interactions avec son environnement ». Dès lors tout est dit, ou presque, sur ce qu'est finalement le système immunitaire: un ensemble complexe d'éléments en équilibre «instable» mais, in fine, terriblement efficace, interagissant avec l'extérieur comme vers l'intérieur, très régulé et répondant à des règles strictes mais adaptatives. Ces règles ne sont pas toutes totalement connues aujourd'hui, l'immunologie demeure encore une science «nouvelle», très dynamique, en continuelle force de découvertes. La complexité du système immunitaire provient du fait qu'il a évolué de telle sorte

Vignette (Photo $\Subset$ CNRS - Fengjuan Wang).

\section{Autophagie, \\ auto-immunité \\ et maladies \\ auto-immunes}

Sylviane Muller ${ }^{1,2}$

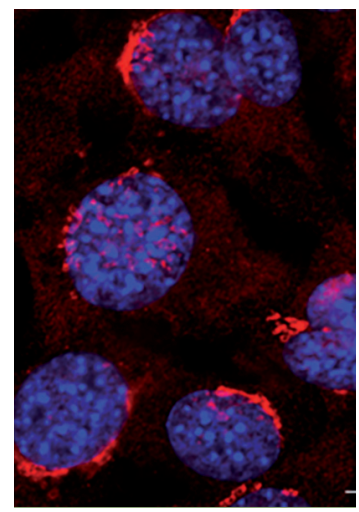

${ }^{1}$ CNRS, Immunopathologie et chimie thérapeutique, Laboratoire d'Excellence Medalis, Institut de biologie moléculaire et cellulaire, 15 , rue René Descartes, 67000 Strasbourg, France; ${ }^{2}$ Institut d'études avancées de l'université de Strasbourg (USIAS), Strasbourg, France. sylviane.muller@unistra.fr

qu'il est censé nous protéger de toutes substances étrangères, même encore inconnues, qui se présenteraient de manière dangereuse aux abords de nos frontières naturelles (peau, muqueuses). Cette remarquable efficacité qui nous protège des infections de toutes sortes - agressions virales, bactériennes, parasitaires, que nous côtoyons en continu dans notre vie - présente quelques revers. Le système immunitaire, aussi finement ciselé qu'il puisse l'être, doit en permanence distinguer le bien du mal, le soi (toléré) de l'étranger, c'est-à-dire du non-soi (non toléré). De là, certaines défaillances peuvent émerger. Pour notre survie, un mécanisme complexe de reconnaissance, mené en plusieurs étapes, avec des systèmes de filtres successifs, s'est élaboré au cours de l'évolution. In fine, chez chacun d'entre nous, un système de tolérance au soi est mis en place vis-à-vis d'antigènes $(\mathrm{Ag})$ donnés ou de certains épitopes présentés par ces $A g$, éléments structuraux linéaires ou conformationnels que l'on peut retrouver sur des formes biochimiques très variées, protéines, lipides, glucides ou carbohydrates complexes, acides nucléiques, et qui peuvent faire l'objet de reconnaissances croisées. Dans un premier temps, l'organisme doit entrer en contact avec l'Ag. Dans un second temps, sa réponse vis-àvis de cet Ag doit être annulée, soit par destruction (apoptose) des lymphocytes qui reconnaissent l'Ag et qui seraient susceptibles de se retourner contre lui, soit par leur neutralisation. Ces mécanismes de tolérance centrale (éducation au niveau thymique des lymphocytes $T$, ou LT, et éducation au niveau de la moelle osseuse des lymphocytes $B$, ou LB) et périphérique (éducation, durant toute la vie, de la maturation des lymphocytes) font intervenir nombre de facteurs qui, par le jeu de ces sélections et éliminations, ne vont laisser subsister à terme que les composés du soi qui vont être «invisibles» et ignorés par le système immunitaire et ne pas donner lieu à une quelconque réaction (anergie). 
Pour être capable de reconnaître tout motif agressif nouvellement généré - une bactérie émergente, un virus exprimant des mutations aléatoires - le système immunitaire s'est doté de mécanismes spécifiques mais néanmoins quelque peu permissifs et versatiles. Cette reconnaissance, dite «dégénérée », de l'Ag par les molécules du complexe majeur d'histocompatibilité $(\mathrm{CMH})$ et sa présentation aux récepteurs des cellules $T$ (TCR), est essentielle dans le processus d'adaptabilité du système immunitaire à tout nouvel envahisseur ou élément antigénique qui se révélerait délétère. Cette tolérance relative de reconnaissance présente cependant un inconvénient qui peut donner lieu à un défaut immunitaire majeur, à savoir un certain degré de réactivité vis-à-vis d'éléments du soi, en principe ignorés. On parle alors d'auto-immunité qui peut, selon les terrains - génétiquement prédisposés, par exemple - donner lieu à une maladie auto-immune (MAI) ${ }^{1}$.

\section{Les maladies auto-immunes}

L'auto-immunité n'est donc pas synonyme de MAl, loin s'en faut. Un ensemble de facteurs intervient dans la survenue d'un syndrome autoimmun. Ces facteurs sont complexes, encore souvent mal identifiés, que ce soit dans les MAI spécifiques d'organes ou dans les MAI systémiques et souvent chroniques. Les MAI sont en général d'origine multigénique, très polymorphes, d'évolutions difficilement prédictibles, dépendantes d'éléments environnementaux (terrain infectieux, déséquilibre du microbiote, habitudes alimentaires, contexte hormonal avec un déséquilibre de prévalence entre femmes et hommes $[60](\rightarrow)$, exposition toxicologique ou aux rayonnements, notamment ultra-violets). L'environnement $\rightarrow$ Voir la Synthèse de N. Dragin et al., $m / s n^{\circ} 2$, février 2017, page 169 social est un élément très influant, tant sur le déclenchement que sur le déroulement de la pathologie. Le tropisme des MAI demeure souvent une énigme sur le plan clinico-biologique. Notre ignorance se révèle également dans notre incapacité à pouvoir prédire les évolutions de la maladie, l'apparition de phases d'exacerbation subites et de rémissions. Relevons ici que les MAl touchent environ $8 \%$ de la population en France. Le lupus érythémateux disséminé (LED) affecte environ 20000 personnes en France (chiffres de 2010) et plus de 5 millions de patients dans le monde. D'autres MAI sont encore plus fréquentes, comme la polyarthrite rhumatoïde (600 000 personnes touchées en France), le diabète de type I (300 000 en France), la maladie de Crohn (ou MC, 200000 en France en 2011) ou la sclérose en plaques (ou SEP, 70 à 90000 en France). Le spectre des MAI est vaste, ciblant un site vital de l'organisme (les cellules $\beta$ productrices d'insuline du pancréas, ce qui conduit à des déficits sévères, dans le diabète de type I ; la gaine de myéline protectrice qui entoure les axones dans le système nerveux central [SNC] dans la SEP; la glande thyroïde dans la

\footnotetext{
${ }^{1}$ Nous possédons tous des autoanticorps (autoAc) et des lymphocytes autoréactifs circulants mais ces éléments sont généralement présents à bas bruit et ne sont pas nocifs. Ce sont, par exemple, des IgM (immunoglobulines $M$ ) d'affinité modérée envers des éléments membranaires, des LB sécrétant des anticorps $(A c)$ naturels autoréactifs ou des LT autoréactifs de faible affinité. II est généralement avancé que cette auto-immunité dite physiologique régule l'homéostasie du système immunitaire. II peut arriver, cependant, que les Ac soient des IgG de plus forte affinité. Certains, alors délétères, seront capables de se déposer dans des organes ciblés (reins, peau, articulations, cerveau, tissus glandulaires), seuls, ou complexés à des autoantigènes (autoAg) spécifiques, les lymphocytes B différenciés en plasmocytes produiront alors des Ac pathogènes.
}

thyroïdite de Hashimoto), ou un ensemble d'organes ou de tissus (les nerfs périphériques dans le syndrome de Guillain-Barré et la polyradiculonévrite aiguë inflammatoire), le tube digestif (de la bouche à l'anus) dans la MC, les articulations dans la spondylarthrite ankylosante, ou encore la presque totalité de l'organisme dans le LED qui est l'archétype des MAl chroniques systémiques. Des pathologies comme le psoriasis, une maladie inflammatoire chronique de la peau qui peut toucher différentes parties du corps (coude, genoux, cuir chevelu, etc.), présentent également des composantes auto-immunes.

La diversité phénotypique que l'on reconnaît dans les manifestations pathologiques de l'auto-immunité reflète aussi une mosaïque de cibles antigéniques dont la liste s'étend continuellement à mesure de l'amélioration de la sensibilité des méthodes de détection biochimique (approches avancées de protéomique, criblages automatisés à grande échelle, dépistage de grandes cohortes de patients). Plus de cent autoAg ont ainsi été identifiés dans le cas du LED. Tous ne sont pas des éléments immunologiquement dominants, parfois ce sont des constituants du soi reconnus de manière très spécifique mais par des anticorps $[\mathrm{Ac}]$ qui sont produits chez quelques patients seulement. Malheureusement, peu de marqueurs de MAI émergent des longues listes de cibles auto-immunes répertoriées ${ }^{2}$. Le lien établi entre ces cibles et la nature de la maladie, son intensité, sa forme, son évolution est souvent ténu. Néanmoins, on notera avec intérêt que certains groupes, ou clusters, de réactivité permettent d'orienter le diagnostic (sans l'asseoir bien entendu, à l'exception des rares marqueurs). À ce jour, il nous est bien difficile de comprendre pourquoi des Ac ciblant la particule Ro (ou SSA) - des anticorps antinucléaires dirigés contre I'une des protéines Ro (Ro52, Ro60) associée aux ARN-Y non codants - apparaissent dans le syndrome de Gougerot-Sjögren (et pourquoi une sous-population d'Ac antiprotéine Ro52 sont liés au lupus néonatal); pourquoi des autoAc réagissant avec la topoisomérase I sont retrouvés dans le sérum de patients atteints de sclérodermie; et pourquoi des Ac anti-transglutaminase (tTG), notamment de classe IgA, caractérisent la maladie cœliaque, une

\footnotetext{
${ }^{2}$ Les nombreux travaux centrés depuis des décennies sur la meilleure définition des épitopes reconnus par les autoAc et l'élucidation des fonctions physiopathologiques de certains de ces autoAc nous ont apporté d'immenses connaissances permettant de classer les MAl, de tenter d'apporter des réponses aux multiples questions posées sur leur origine, leur mode d'action et de les « apprivoiser » pour qu'ils nous servent d'outils de diagnostic et de suivi thérapeutique. Les marqueurs reconnus, validés par les agences administratives américaines et européennes et acceptés dans l'usage courant en laboratoire demeurent cependant encore peu nombreux et pour certains insuffisamment représentés en terme de fréquence. Nous citerons les Ac anti-ADNdb (double brin) et anti-Smith (qui ciblent les petites ribonucléoprotéines nucléaires ou small nuclear ribonuclear protein [snRNP] dans le LED), anti-tTG (transglutaminase) dans la maladie cœliaque, anti-glutamate acide décarboxylase dans le diabète de type $\mathrm{I}$, ce dernier étant un bon marqueur de dépistage avec une prévalence d'environ $80 \%$ mais à la valeur prédictive d'évolution faible. Quelques autres ont été décrits.
} 
maladie intestinale inflammatoire chronique auto-immune induite par la gliadine ${ }^{3}$. D'immenses avancées ont pourtant fait progresser nos connaissances de manière sensible. Ainsi, la cible des Ac anti-phospholipides qui sont associés à des manifestations thrombotiques et obstétricales dans le syndrome dit des Ac anti-phospholipides a été particulièrement étudiée. II s'agit de protéines intervenant dans la cascade de la coagulation ou de la fibrinolyse comme la $\beta 2$ - glycoprotéine, la prothrombine, la protéine $C$ activée, la protéine $S$ ou l'annexine V. L'annexine V présente une fonction anticoagulante. Elle empêche la prothrombine de s'activer à la surface des membranes des villosités placentaires. Dans le cas de la neuromyélite optique de Devic, considérée comme un spectre de maladies inflammatoires démyélinisantes du système nerveux central (SNC), des auto-Ac ciblent un canal à eau transmembranaire, l'aquaporine 4, une protéine jouant un rôle central dans le maintien du volume d'eau du cerveau. La liaison de ces Ac à leur cible entraîne une inflammation, une détérioration du canal et une irritation des tissus cérébraux avoisinants. Citons enfin les Ac spécifiques du nucléosome, une structure fondamentale de la chromatine, très organisée et étroitement conservée, constituée d'histones et d'ADNdb (ADN double brin). Si les Ac anti-histones sont retrouvés dans nombre de situations pathologiques, auto-immunes, infectieuses ou cancéreuses, de nombreuses études ont démontré le rôle de modifications post-traductionnelles variées et décisives dans ces protéines qui les rendent uniques et, de fait, font des Ac qui les reconnaissent des marqueurs potentiellement très utiles [1-3]. À ce stade, de nombreuses questions se posent. La grande majorité des cibles reconnues par les acteurs humoraux et cellulaires auto-immuns sont des éléments intracellulaires, enfouis, normalement inaccessibles au sein d'organes et tissus. Ce sont souvent des épitopes contenus dans des protéines partenaires d'acides nucléiques, organisées dans des assemblages macromoléculaires multimériques complexes, associant co-facteurs et ligands (comme les splicéosomes ${ }^{4}$, les nucléosomes, les ribosomes). Pourquoi ces épitopes sont-ils reconnus par les autoAc? Sont-ils à l'origine des autoAc que nous détectons dans le sang périphérique? De toutes ces études, qu'avons-nous appris de leur exposition, sans doute anormale, à l'origine de leur «visibilité »? Maintes théories ont été proposées dont certaines ont été très documentées expérimentalement au cours des dizaines d'années durant lesquelles ces questions majeures ont été posées. Les phénomènes de mimétisme moléculaire entre agents infectieux et $\mathrm{Ag}$ du soi ou ceux mettant en jeu les processus d'apoptose affectant la morphologie cellulaire et exposant des motifs antigéniques normalement cachés (cryptiques) ont ainsi prévalu. Une littérature approfondie a en effet été publiée et des faisceaux très forts d'arguments ont été avancés dans ces directions. La théorie éphémère de diversification antigénique, aussi dite de diffusion du répertoire de proche en proche, énoncée par Eli Sercarz [4], a également fait l'objet d'études avancées [5-7]. Elle revient aujourd'hui dans les idées qui concourent au développement de stratégies thérapeutiques, notamment dans la SEP. Face à ces éléments et concepts, posons-nous aujourd'hui la question des relations entre auto-immunité et autophagie.

${ }^{3}$ La gliadine est la fraction protéique du gluten que l'on retrouve dans le blé.

${ }^{4}$ Le splicéosome est un complexe ribonucléoprotéique qui participe à l'épissage des ARN pré-messagers.

\section{Auto-immunité et autophagie}

L'autophagie est un mécanisme cellulaire qui consiste en la dégradation de constituants du cytoplasme de la cellule utilisant ses propres lysosomes. Il s'agit d'un processus cellulaire vital qui est catabolique mais qui favorise aussi la survie des cellules en conditions de stress, comme par exemple, celles résultant d'une carence en acides aminés. Ce mécanisme finement régulé permet la dégradation par une cellule de ses constituants cytoplasmiques, inutiles, mal formés ou agrégés et donc délétères, dans des vésicules à double membrane fusionnant à terme avec des lysosomes et aboutissant à une production rapide d'ATP (Figure l). La formation de ces vésicules appelées autophagosomes requiert un ensemble de gènes, les gènes ATG (autophagy-related genes), dont l'action très régulée s'égrène dans un programme complexe finement ordonné $[8-10](\rightarrow)$.

$(\rightarrow)$ Voir la Synthèse de J. Arnold et al., $\mathrm{m} / \mathrm{s} \mathrm{n}^{\circ} 3$, mars 2016, page 281

Notons que certains de ces gènes interviennent dans d'autres mécanismes cellulaires que ceux de l'autophagie.

Au-delà de son rôle protecteur lors de stress énergétiques, l'autophagie est impliquée de manière décisive dans la régulation de l'immunité, en particulier dans le développement, l'éducation et l'homéostasie des lymphocytes [11] ainsi que lors de la présentation antigénique. Divers modèles murins d'invalidation conditionnelle sont venus étayer le rôle primordial de plusieurs gènes liés à l'autophagie dans la fonction des LT, les données générées dans les LB demeurant encore complexes à analyser à ce jour [12-18]. Différents gènes autophagiques arbitrent en effet la qualité des fonctions des thymocytes. Des polymorphismes dans certains de ces gènes, comme ATG5 et DRAMI (DNA-damage-regulated autophagy modulator 1), impliqués respectivement dans l'exécution et l'induction de l'autophagie, ont été corrélés au développement du lupus $[19,20]$. D’autres altérations de gènes autophagiques ont également été impliquées comme par exemple l'identification récente d'un locus à risque concernant $A T G 16 L 2$ dans une population coréenne de 1174 patients lupiques et 4246 individus témoins [21]. Ce locus représente aussi un facteur de risque dans une population de patients européens atteints de MC. Des hypothèses longtemps restées sans démonstration cellulaire et immunologique liant autophagie et auto-immunité ont ainsi été formulées mais ce n'est qu'en 2012 que les premières études expérimentales concernant le lupus ont été publiées démontrant l'existence de défauts cellulaires dans la 
A

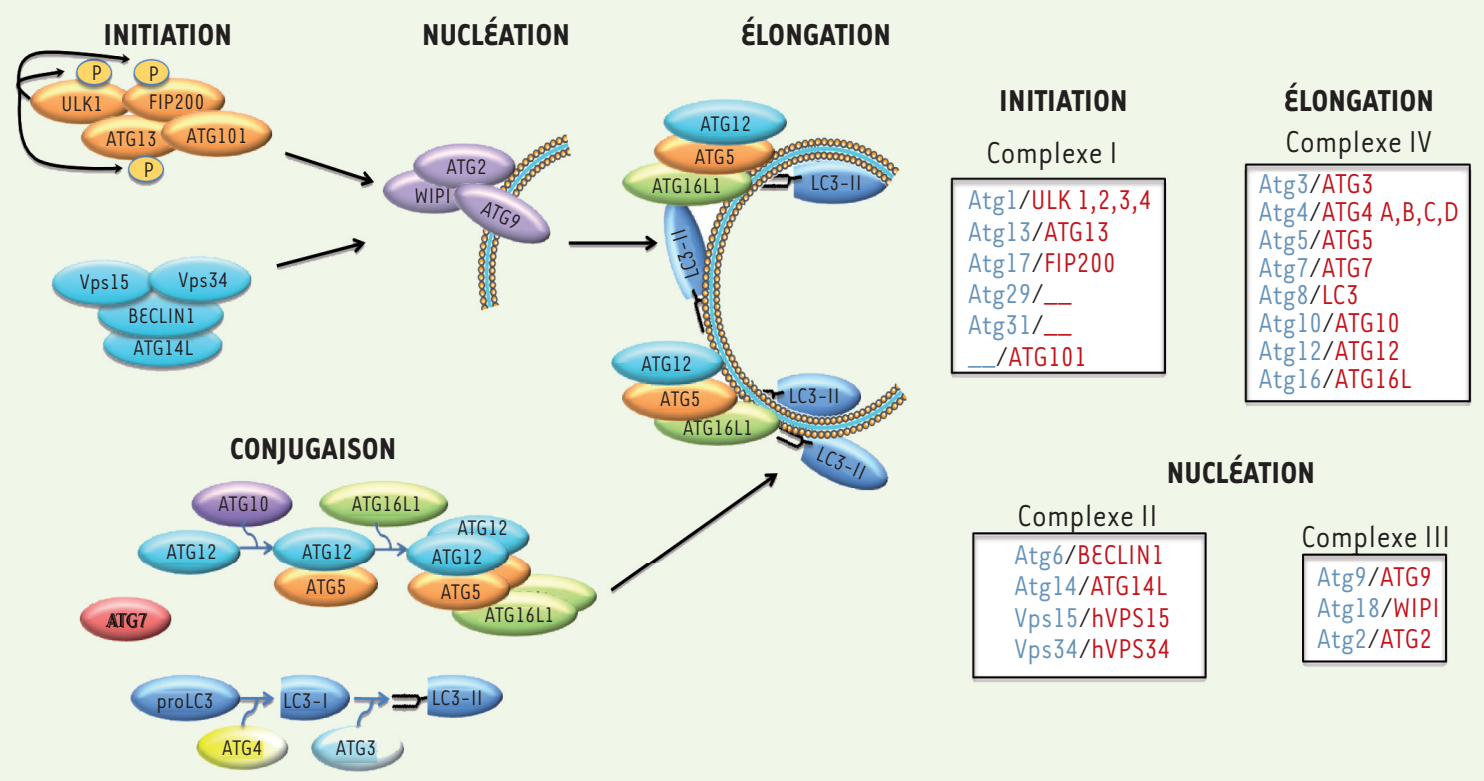

B

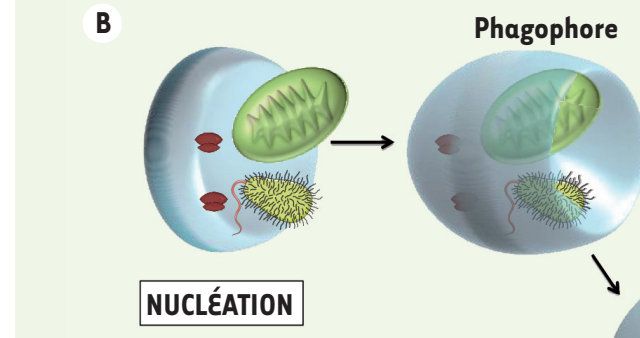

Phagophore

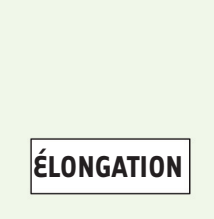

Autophagosome
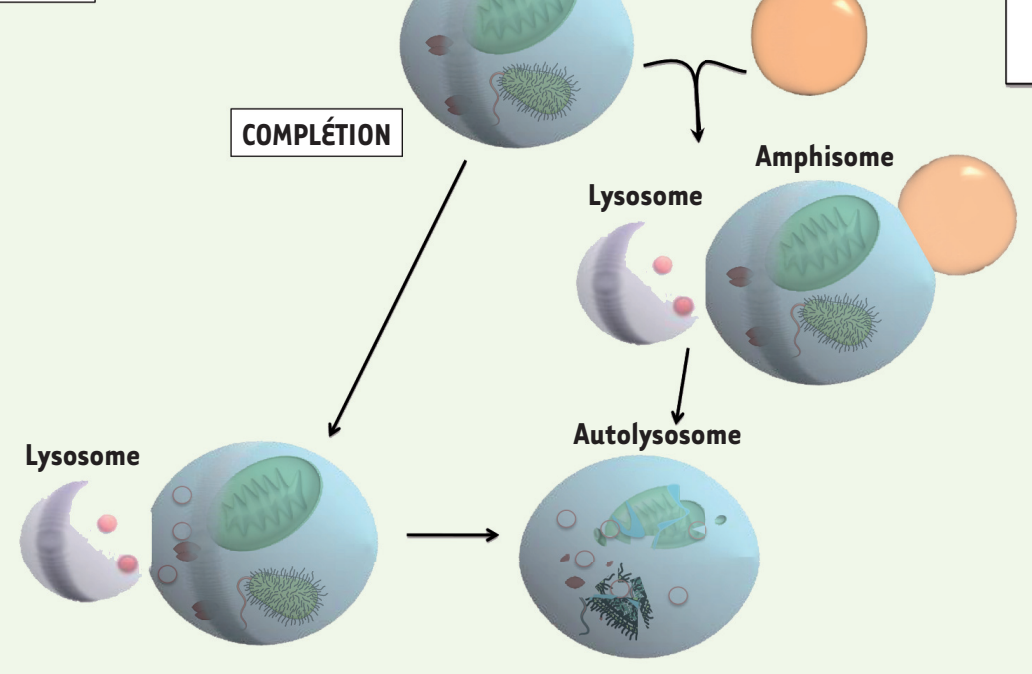

Figure 1. Formation des autophagosomes et autolysosomes, vésicules stratégiques dans l'apprêtement antigénique. A. Représentation des protéines impliquées dans la formation de l'autophagosome. Les complexes ULK (Unc-51-like autophagy-activating kinase) et VPS34 qui respectivement présentent une activité kinase et phosphoinositide 3 kinase sont nécessaires à l'initiation du processus de macroautophagie. Ils permettent le recrutement des complexes ATG (autophagy related gene)5/ATG12/ATG16L et MAP1LC3-II (microtubule-associated protein light chain 3) issus du système de conjugaison, nécessaires à l'élongation du phagophore. Encarts : liste des protéines impliquées dans chaque étape de la formation de l'autophagosome (en bleu, les protéines identifiées chez la levure; en rouge, celles retrouvées chez les mammifères). Un certain nombre de ces protéines sont conservées au cours de l'évolution (voir Glossaire). B. Vue générale de la voie macroautophagique chez les mammifères. Le phagosome formé peut séquestrer divers organites intracellulaires et des agents microbiens présents puis, par fusion avec le lysosome, dégrader ces éléments qui vont fournir des nutriments à la cellule. 


\section{GLOSSAIRE}

Ac : anticorps

ADA : anti-drug antibody (anticorps anti-médicament)

ADN : acide désoxyribonucléique

$\mathrm{Ag}$ : antigène

ATP : adénosine triphosphate

$B C R$ : récepteur des lymphocytes $B$

CMA : chaperone-mediated autophagy (autophagie relayée par les chaperonnes)

CMH : complexe majeur d'histocompatibilité

CPA : cellule présentatrice d'antigènes

$\mathrm{db}$ : double brin

DC : cellules dendritiques

FDA : Food and Drug administration

HSP : heat shock protein

Ig: immunoglobulines

LAMP : lysosomal-associated membrane protein

LAP : LC3-associated phagocytosis

LB : lymphocyte B

LED : lupus érythémateux disséminé

LT : lymphocyte T

MAI : maladie auto-immune

MAPILC3 (ou LC3) : microtubule-associated protein light chain 3

MC : maladie de Crohn

MRL/Ipr: Murphy roths large/lymphoproliferation

mTOR : mammalian target of rapamycin

NZB : New Zealand black

NZW : New Zealand white

SNC : système nerveux central

SEP : sclérose en plaques

SPSTM1 : séquestosome 1

TCR : récepteur des lymphocytes T

TLR : toll-like receptor l'autophagie relayée par les chaperonnes (CMA $)^{5}$ [27] ont également été révélés dans le lupus [28]. II a notamment été découvert que l'expression des marqueurs de CMA, LAMP2A (lysosome-associated membrane protein 2) et $\mathrm{HSP}$ (heat shock protein)A8/HSC70, était fortement augmentée dans les LB des souris MRL/lpr. Cette surexpression des protéines LAMP2A et HSPA8 dans les LB a pu être reliée au niveau transcriptionnel, dans la rate (pour LAMP2A) et les ganglions lymphatiques (pour LAMP2A et HSPA8), mais pas dans le thymus et la moelle osseuse des souris MRL/Ipr (Wang et Muller, données non publiées). L'autophagie est essentielle pour la présentation antigénique des peptides aux LT dans le contexte des molécules du CMH. Elle intervient à plusieurs niveaux dès les stades des sélections positive et négative qui assurent la tolérance au soi $[15,29]$. Dans ce processus, ATG5 et d'autres éléments du processus (macro)autophagique seraient impliqués. Au niveau du thymus, les cellules épithéliales et les cellules dendritiques expriment naturellement une forte activité autophagique. Parmi les différentes implications de l'autophagie dans la survie et l'activation des lymphocytes, relevons qu'elle favorise la présentation d'Ag cytosoliques, donc du soi [30], tout comme celle d'Ag viraux intracellulaires par les molécules du CMH-II [31]. Bien que le mécanisme de cette présentation reste encore à étayer in vivo, des arguments expérimentaux semblent indiquer que l'autophagie pourrait, par ce mécanisme, grandement influencer l'activation des LB autoréactifs. Au travers des voies macroautophagiques et de la CMA, toutes deux étroitement reliées au lysosome, il est proposé qu'en amont, l'autophagosome délivre des peptides endogènes, notamment des peptides du soi clivés par les cathepsines actives en milieu acide, aux molécules du CMH-II présentes dans le compartiment endosomal tardif MIIC (Figure 2). C'est dans ce compartiment, une structure lamellaire observable par microscopie électronique, que se déroule l'essentiel du chargement des molécules de classe II. Les Ag ainsi capturés et apprêtés sont ensuite présentés à la surface des

\footnotetext{
${ }^{5}$ La macroautophagie (Figure 1B) et la CMA sont les deux formes autophagiques les plus étudiées à ce jour et de fait les mieux appréhendées. La CMA est une voie sélective d'autophagie permettant la translocation de substrats protéiques cytosoliques à travers la membrane du lysosome. Les protéines importées sont ensuite dégradées dans la lumière du lysosome par les protéases luménales. La CMA est une voie plus sélective que la macroautophagie; elle cible les substrats possédant un motif peptidique particulier, de type KFERQ-like qui est reconnu par la protéine chaperonne HSPA (heat shock $70 \mathrm{kDa}$ protein 8 ). Cette dernière facilite l'import du substrat protéique dans le lysosome non seulement en interagissant avec le récepteur LAMP2A (lysosomal-associated membrane protein 2A) lysosomal, mais aussi en participant au dépliement du substrat ciblé. Des expériences d'immunoprécipitation ont montré que HSPA8 associée à la membrane lysosomale, interagit avec la chaperonne HSP90 et les co-chaperonnes HIP, HOP, BAG-1 et HSP40. Chacune de ces protéines est nécessaire à l'importation du substrat dans le lysosome. La CMA est présente à l'état basal dans les cellules de mammifères et son activité est fortement stimulée par de nombreux signaux de stress tels que la carence en acides aminés ou un stress oxydatif.

voie macroautophagique des LT chez l'homme et la souris, puis ultérieurement dans les LB [22-24]. La littérature demeure cependant encore assez confuse vis-à-vis de ces défauts qui, par exemple dans le lupus, relate selon les études un état d'activation ou d'inhibition du flux macroautophagique. Ces divergences relèvent de plusieurs facteurs fondamentaux et expérimentaux. Les résultats semblent en effet souffrir d'être différemment appréciés et interprétés. Tant chez lement varier selon l'état de la cellule, dépendant de son contexte inflammatoire, notamment cytokinique, plus ou moins amplifié sensibles selon le modèle murin de lupus spontané considéré, à savoir les souris MRL/MpJ-Fas'pr (Murphy roths large/lymphoproliferation) que nous abrégerons MRL/Ipr et les souris (NZBxNZW) (New Zealand black $x$ New Zealand white) Fl qui ne présentent pas les mêmes défauts physiopathologiques intrinsèques. La méthodologie utilisée pour démontrer les défauts éventuels des circuits autophagiques est également capitale comme l'indiquent plusieurs études [25, 26]. Récemment des défauts majeurs dans la voie de 
cellules présentatrices d'Ag, notamment les cellules dendritiques et les LB. Ils seront reconnus finalement par le TCR des LT autoréactifs (dans le cas d'Ag du soi) [32-34]. On l'aura compris, l'état de maturation des autolysosomes ainsi que la densité de lysosomes «CMA-compétents » seront déterminants dans ce mécanisme intracellulaire. Les récepteurs de type Toll-like (TLR) jouent un rôle régulateur important [35] dans ce processus tout comme l'activité protéasique des vésicules lysosomales et endosomales, finement régulée, qui dépend du pH [36]. Un rôle régulateur est joué par l'ubiquitination de la chaîne bêta de certaines molécules de classe II après chargement des peptides ${ }^{6}[37,38]$.

D’autres mécanismes sont impliqués dans la présentation antigénique comme celui connu sous le nom de phagocytose associée à MAPILC3 (ou LC3) appelée LAP (LC3-associated phagocytosis) (Figure 2) [39, 40] qui agit comme un activateur ou, au contraire, comme un stabilisateur de la présentation antigénique selon les cellules présentatrices considérées, cellules dendritiques ou macrophages. La LAP exploite une partie de la machinerie autophagique. Elle n'implique pas les éléments du processus canonique (Figure 1), notamment le complexe de pré-initiation contenant ULKl (Unc-51-like autophagy activating kinase 1) et FIP200 (FAK family kinase-interacting protein of $200 \mathrm{kDa}$, aussi connu sous le nom de RBICCl, RBI-inducible coiled-coil 1). À la place, la LAP fonctionne en utilisant un complexe formé de la Beclin-1 et VPS34 (PIK3C3), sans ATG14. La LAP pourrait être, davantage encore que l'autophagie canonique, déterminante dans la réponse lupique, en relation avec les défauts d'élimination qui ont été identifiés depuis des décennies dans cette pathologie [41]. Bien qu'un important travail reste à mener pour démontrer l'implication directe de la LAP chez les patients lupiques, des données génétiques semblent étayer cette possibilité [42].

Chez la souris lupique MRL/Ipr, des défauts affectant les lysosomes sont retrouvés [28]. Pour des raisons qui restent à élucider, le $\mathrm{pH}$ moyen des lysosomes dans les LB spléniques de ces souris est augmenté par rapport au $\mathrm{pH}$ des lysosomes de souris saines. D'autres défauts qui affectent différents éléments des voies autophagiques sont observés chez ces souris. Deux marqueurs de la CMA dont le rôle est déterminant dans la présentation antigénique, LAMP2A et HSPA8, sont surexprimés et le flux autophagique est accru $[28,43]$ (et données non publiées). Le nombre de vacuoles autophagiques qui s'accumulent dans les LT est également augmenté [22]. Des études systématiques et intégrant davantage d'échantillons seront nécessaires pour tenter d'établir des corrélats métaboliques et physiopathologiques impliquant des déficits ou au contraire des activations de l'activité autophagique, en distinguant divers modèles murins pathologiques, induits ou spontanés, de MAI, l'état d'avancement de la maladie, divers organes et tissus. Diverses méthodologies devront être appliquées pour rendre les données robustes et fiables. Aujourd'hui, très peu de données expérimentales sont disponibles et nous ne pouvons pas encore appréhender les conséquences des dérèglements affectant ces processus, ou réellement établir le lien pressenti entre apoptose et autophagie, qui pourrait être particulièrement décisif comme cela

${ }^{6}$ L'enzyme engagée dans cette étape est une ligase $\varepsilon 3, \mathrm{MARCHl}$, qui intervient également dans l'ubiquitination du récepteur de la transferrine, de CD86 (exprimé à la surface des cellules présentatrices, délivrant des signaux de co-stimulation nécessaires à l'activation et la survie des LT) et de FAS (protéine transmembranaire pouvant favoriser la mort par apoptose de LT activés), par exemple. apparaît, par exemple, dans le cancer. Notons, enfin, que l'absence d'activateurs et d'inhibiteurs pharmacologiques efficaces in vivo, et surtout sélectifs, représente un frein non négligeable aux avancées dans les connaissances dans ce domaine [17, 44-47].

\section{Le peptide P140, un outil pharmacologique et une arme thérapeutique}

Le peptide P140 qui cible certains circuits de signalisation autophagique est un candidat-médicament très prometteur. II correspond à la séquence 131-151 de la protéine splicéosomale U1-70K qui est fréquemment reconnue par des autoAc et par les LT dans le lupus. Ce peptide possède en position 140 un résidu de phosphosérine introduit chimiquement au cours de sa synthèse en phase solide [48]

L'étude du mécanisme d'action du P140, exploré en première intention chez la souris lupique MRL/Ipr, a montré qu'il se lie à la protéine HSPA8 [50] qui a un rôle central dans la CMA et est surexprimée dans le lupus. Après administration intraveineuse du peptide (à raison de trois injections, voire une seule pour la mesure de certains paramètres, de $100 \mu \mathrm{g}$ de peptide par souris), toute une série d'anomalies cellulaires et moléculaires liées à la pathologie lupique n'étaient plus observées: c'est le cas de la surexpression de HSPA8, de LAMP2A et des molécules du CMH-II [28, 43]. In vitro, un effet direct du P140 sur la CMA a été mis en évidence ${ }^{8}$ [28]. En se liant à HSPA8, le peptide $\mathrm{P} 140$ pourrait donc atténuer l'activité de la CMA et ainsi affecter indirectement la macroautophagie. Après injection intraveineuse à des souris MRL/lpr, le peptide pénètre dans les LB en utilisant la voie des clathrines et la voie endo-lysosomale. II se retrouve alors dans le lumen des lysosomes. Dans ces vésicules acides, en interagissant avec l'HSPA8 lysosomale, le peptide P140 pourrait déstabiliser l'hétérocomplexe HSPA8-HSP90 entouré de ses ligands et corécepteurs et/ou LAMP2A, comme cela a été démontré $[27,28]$, ce qui perturberait la CMA et la macroautophagie, moins sélective que la CMA. Après administration du peptide P140 aux souris lupiques, une accumulation de protéines SDSTM1/p62 (sequestosome 1) et MAPILC3 est observée, suggérant un ralentissement du flux autophagique [43] (et données non publiées). Bien que nombre d'éléments nécessiteraient d'être démontrés plus avant in vivo, il semble que la déstabilisation du complexe HSPA8-HSP90, via celle de LAMP2A, et le ralen-

\footnotetext{
${ }^{7}$ Nous avons découvert plus tard que ce remplacement ponctuel correspond à une modification post-traductionnelle naturelle de la protéine qui s'opère en début du processus apoptotique alors que la protéine U1-70K, qui est normalement hautement phosphorylée, est ainsi déphosphorylée par un système de phosphatases impliquant notamment la protéine phosphatase 2A (PP2A) [49].

${ }^{8}$ Collaboration avec le laboratoire d'Ana Maria Cuervo (Albert Einstein College of Medicine, Ny, États-Unis)
} 


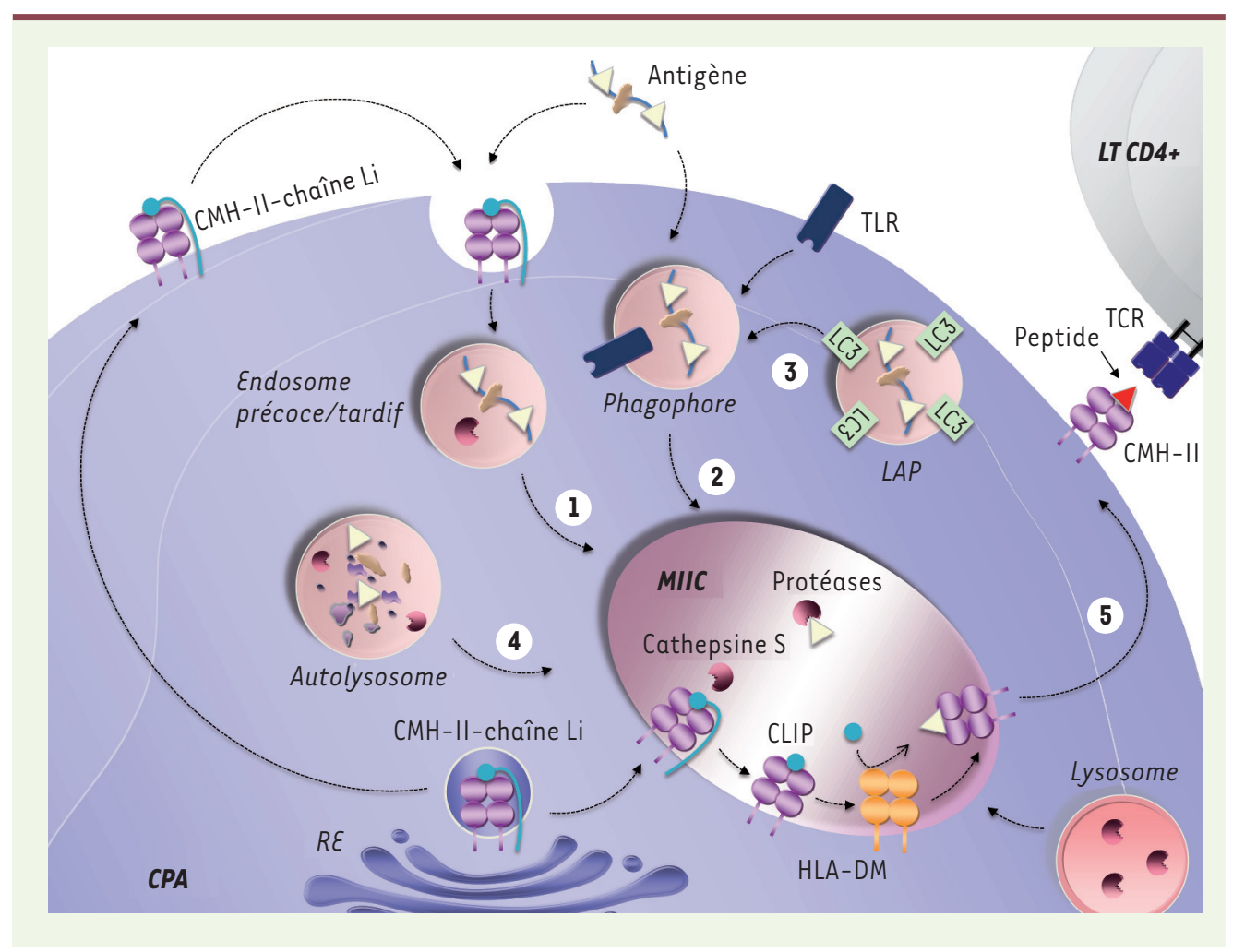

Figure 2. Implication des voies de l'autophagie dans la présentation antigénique par les molécules du CMH-II (complexe majeur d'histocompatibilité de classe II). L'apprêtement et la présentation de l'Ag (antigène) dans les cellules présentatrices d'antigènes (CPA) impliquent le transport de celui-ci à travers la voie endolysosomale où il est dégradé (1). L'Ag peut également être internalisé par phagocytose et se retrouver dans les phagosomes (2). L'utilisation de cette voie peut être facilitée par l'activation des TLR (Toll-like receptor). Le recrutement des protéines MAPILC3 (également appelées LC3 microtubule-asso-

ciated protein light chain 3) au niveau des phagophores induit la dégradation de l'Ag, c'est le phénomène de LAP (LC3-associated phagocytosis) (voie non canonique (3)). Enfin, l'autophagie contribue également à la génération de peptides antigéniques pour leur présentation par le CMH-II (4). C'est au sein du compartiment MIIC que se fait l'apprêtement de l'Ag. Les molécules du CMH-II qui sont synthétisées au niveau du réticulum endoplasmique (RE) forment un complexe avec la chaîne invariante Li. Ce complexe est transporté du Golgi vers les compartiments MIIC, soit directement, soit via la membrane plasmique. La chaîne invariante Li est dégradée dans les MIIC par la cathepsine S. Le fragment CLIP (class IIassociated invariant chain peptide) reste au niveau de la poche peptidique des molécules du CMH-II et son échange avec le peptide antigénique s'opère à l'aide de la molécule HLA (human leukocyte antigen)-DM qui agit comme un véritable catalyseur. À l'issue de ce cheminement qui emprunte diverses voies possibles dont celles de l'autophagie, les molécules du CMH-II chargées sont transportées vers la membrane plasmique pour y présenter les peptides antigéniques aux TCR, récepteurs des lymphocytes T (LT) CD4+ (5).

tissement du flux macroautophagique excessif altèrent le chargement des peptides antigéniques sur les molécules du CMH-Il et leur présentation au TCR des LT. Nous utilisons à dessein le terme «altérer » car le nombre de peptides présentés dans le contexte du CMH pourrait ne pas changer de manière significative. Par contre leur séquence pourrait varier si l'activité des cathepsines est ramenée à un niveau basal. Des études réalisées sur des lysosomes purifiés de LB isolés de modèles lupiques et des analyses de protéomique quantitative permettant d'identifier des différences dans le pool de peptides présentés sont nécessaires afin de mieux comprendre ces différents aspects [51-53].

$\varepsilon$ aval, les effets de la déstabilisation des molécules de $\mathrm{CMH}-\mathrm{Il}$ et du défaut de présentation de peptides endogènes autoantigéniques au TCR des LT autoréactifs sont décisifs (Figure 2). Ils affectent en effet la signalisation des LT et leur rôle accessoire pour les LB autoréactifs. La différenciation des LB en plasmocytes peut ainsi être atténuée et le taux d'autoAc sécrétés dans le sang périphérique abaissé, notamment les anti-ADNdb. Gageons que c'est via les voies autophagiques, notamment la macroautophagie et la CMA qui traitent les Ag intracellulaires, que des séquences normalement enfouies dans des éléments du soi sont in fine présentées aux $\mathrm{LT} \mathrm{CD4} 4^{+}$dans le contexte de molécules du CMH-II. Serait ainsi partiellement levée la question, que nous nous nous posons depuis plusieurs décennies, de savoir comment des Ag du soi normalement inaccessibles sont reconnus par les lymphocytes auto-immuns. Certains de ces effets, notamment l'extinction de la réponse $T$ vis-à-vis d'épitopes du complexe splicéosomique, ont été démontrés après administration du peptide P140 à des souris MRL/Ipr [7, 54]. La survie des souris traitées est très significativement prolongée, les lésions cutanées ne sont plus apparentes et les atteintes des vaisseaux sanguins et des reins sont très nettement réduites $[43,48]$.

Ces résultats, obtenus chez la souris MRL/lpr, suggèrent que le peptide P140 affecterait la présentation d'Ag endogènes par les molécules du CMH-II en inhibant les différentes voies autophagiques qui s'expriment de manière exagérée et en altérant leur clivage au sein du lysosome. 
Les perturbations typiques ne sont plus retrouvées ou apparaissent de manière très retardée. Cela concerne notamment l'envahissement des organes lymphoïdes et du sang périphérique par une population de LT double négatifs $\left(C D 3^{+} C D 4^{-} C D 8^{-} \operatorname{CD} 45 R / B 220^{+}\right)$, des défauts de trafic cellulaire, d'expression de marqueurs d'activation des LT et des LB et des cytokines circulantes. Certains réarrangements des TCR et $B C R$, spécifiques des souris MRL/lpr, ne sont plus retrouvés après leur traitement par le P140 [54]. Tous ces effets n'affectent pas la réponse $B$ et $T$ des souris soumises à une infection virale [7] et n'a que peu de conséquences sur la réponse contre un immunogène T-dépendant étranger comme l'ovalbumine [54]. Ces résultats sont d'une extrême importance. Ils indiquent que le peptide P140 n'est pas immunosuppresseur (la diversité des répertoires TCR et BCR est d'ailleurs maintenue après traitement), qu'il n'agit pas sur la globalité du système immunitaire mais qu'il intervient de manière ciblée sur la réponse aux $\mathrm{Ag}$ du soi, sans affecter la réponse envers des $\mathrm{Ag}$ du non-soi. L'efficacité du peptide P140 a été évaluée chez des patients lupiques. Après l'obtention de résultats très encourageants observés lors des phases cliniques préliminaires (phases $\|$ a et $\mid l b$ ), des essais cliniques avancés de phase III, exploitant le peptide P140 (Lupuzor ${ }^{\mathrm{TM}}$ ) sont actuellement en cours aux États-Unis, en Europe et dans certaines régions de l'Océan Indien. Comme nous l'avions montré chez la souris MRL/Ipr, qui, rappelons-le, est un modèle très agressif de lupus, le peptide $\mathrm{P} 140$ a prouvé sa grande efficacité à modérer la maladie chez les patients et ceci sans générer d'effets secondaires délétères qui représentent le principal problème posé par les traitements immunosuppresseurs actuels [55]. Le tréhalose, un sucre «accélérateur» de la macroautophagie agissant indépendamment de la voie mTOR (mammalian target of rapamycin) $[56,57]$, neutralise l'effet bénéfique du P140 [58]. Dans sa formulation thérapeutique, le P140 n'est pas immunogène chez la souris ou les patients [59], un atout considérable sur nombre d'Ac thérapeutiques qui, à terme, génèrent des $A c$ anti-Ac (ou ADA). La FDA (food and drug administration) a accordé la désignation «Fast Track» au dossier Lupuzor ${ }^{T M}$ pour le traitement du LED. Cette procédure permettra d'accélérer le développement clinique du peptide et donc sa future mise sur le marché dès l'issue de la phase III d'essai clinique.

\section{Conclusion}

L'autophagie est un processus physiologique fondamental de l'homéostasie cellulaire. Ce mécanisme hautement régulé s'est révélé être activé ou ralenti de manière pathologique, de façon complexe, selon l'organe ou la cellule. De nombreux efforts seront nécessaires pour comprendre les événements moléculaires et cellulaires à l'origine des dysfonctionnements que l'on observe dans diverses pathologies, notamment l'auto-immunité. D'ores et déjà, il semble patent que moduler l'excès d'autophagie permet d'améliorer l'état clinique des patients chez lesquels un défaut de ce processus est observé. De fait, des recherches immunologiques et pharmacologiques sont entreprises activement afin de développer des outils spécifiques (Ac, peptides, petites molécules) qui soient ciblés sur les voies autophagiques et dépourvus d'effets secondaires [17, 47]. Certaines molécules déjà utilisées en clinique possèdent en partie ces qualités, comme l'hydroxychloroquine qui présente malheureusement des effets non désirés importants. Le peptide P140 semble présenter les caractéristiques requises pour servir de futur médicament. II représente également un outil indéniable pour les études fondamentales visant à explorer la CMA ainsi que l'activité lysosomale dans différents types cellulaires, en interaction avec d'autres mécanismes de vie et mort comme l'apoptose ou les modes de régulation de type protéasome. Manipuler l'autophagie et analyser les effets générés dans divers systèmes demeure aujourd'hui à l'origine d'une source d'informations inédites qui nous feront certainement appréhender nombre d'éléments moléculaires et cellulaires encore inconnus. $\diamond$

\section{SUMMARY}

Autophagy, autoimmunity and autoimmune diseases

Autoimmune diseases such as systemic lupus, are the consequence of immunity directed against the organism itself. The immune system abnormally recognizes self-components as foreign and produces antibodies targeting normal cells and tissues. We and others have discovered a number of failures affecting autophagy pathways in the lymphocytes of model mice and patients with lupus. While the current treatments are mainly based on immunosuppressive drugs that can lead to important side effects, the synthetic phosphopeptide P140/Lupuzor, which targets chaperone-mediated autophagy and displays no side effects, holds a lot of promise as a drug candidate. P140, which is currently evaluated in a phase III clinical trial, targets chaperonemediated autophagy that is hyperactivated in lupus mice, and reduces antigenic peptides presentation to autoreactive helper T cells. Remarkably, we showed in lupus mice that upon treatment with P140, a number of immunological abnormalities affecting the pool of $T$ and $B$ cells as well as many biological and clinical features no longer occur. $\diamond$

\section{REMERCIEMENTS}

Je remercie l'ensemble de l'équipe - chercheurs, doctorants, postdoctorants, ingénieurs et techniciens - qui a généré les résultats sur le peptide P140 décrits dans cette revue, Florent Arbogast pour la composition de la Figure 1, Maud Wilhelm pour la Figure 2, Fengjuan Wang pour l'image montrée en vignette, ainsi que Frédéric Gros pour la relecture de cette synthèse. Les recherches de notre laboratoire sont soutenues financièrement par le CNRS, la Région Grand-Est, le laboratoire d'Excellence Centre de recherche du Médicament Medalis (ANR-10LABX-0034), le programme EquipEx I2MC (ANR-11-EPPX-022), l'Institut d'Études Avancées de l'IdEx Université de Strasbourg (USIAS), ainsi que par ImmuPharma France.

\section{LIENS D'INTÉRÊT}

L'auteur déclare n'avoir aucun lien d'intérêt concernant les données publiées dans cet article. 


\section{RÉFÉRENCES}

1. Van Bavel CC, Dieker JW, Kroeze Y, et al. Apoptosis-induced histone $\mathrm{H} 3$ methylation is targeted by autoantibodies in systemic lupus erythematosus. Ann Rheum Dis $2011 ; 70: 201-7$.

2. Dieker JW, Iglesias-Guimarais V, Décossas $M$, et al. Early apoptotic reorganization of spliceosomal proteins involves caspases, CAD and rearrangement of NuMA. Traffic $2012 ; 13: 257-72$.

3. Muller S, Radic M. Oxidation and mitochondrial origin of NET DNA in the pathogenesis of lupus. Nature Med 2016 ; 22 : 126-7.

4. Lehmann PV, Forsthuber T, Miller A, Sercarz $\varepsilon$. Spreading of T-cell autoimmunity to cryptic determinants of an autoantigen. Nature $1992 ; 358: 155-7$.

5. Vanderlugt CL, Miller SD. Epitope spreading in immune-mediated diseases : implications for immunotherapy. Nat Rev Immunol $2002 ; 2$ : 85-95.

6. Monneaux F, Muller $S$. Epitope spreading in systemic lupus erythematosus : identification of triggering peptide sequences. Arthritis Rheum $2002 ; 46: 1430-8$.

7. Monneaux F, Parietti V, Briand JP, Muller S. Importance of spliceosomal RNP1 motif for intermolecular T-B cell spreading and tolerance restoration in lupus. Arthritis Res Ther $2007 ; 9$ : R111.

8. Feng Y, He D, Yao Z, Klionsky DJ. The machinery of macroautophagy. Cell Res $2014 ; 24$ : 24-41.

9. Subramani S, Malhotra V. Non-autophagic roles of autophagy-related proteins. EMBO Rep 2013 ; $14: 143-51$.

10. Arnold J, Murera D, Arbogast F, et al. L'autophagie et l'homéostasie des LT et B : bien recycler pour un développement durable. Med Sci (Paris) 2016 ; 32 : 281-89.

11. Valdor R, Mocholi $\varepsilon$, Botbol $Y$, et al. Chaperone-mediated autophagy regulates T cell responses through targeted degradation of negative regulators of T cell activation. Nat Immunol 2014; 15 : 1046-54.

12. Botbol Y, Guerrero-Ros I, Macian F. Key roles of autophagy in regulating T-cell function. Eur J Immunol 2016 ; 46 : 1326-34.

13. Pua HH, Dzhagalov I, Chuck $M$, et al. A critical role for the autophagy gene Atg5 in T cell survival and proliferation.J Exp Med 2007 ; $204: 25-31$.

14. Stephenson LM, Miller BC, Ng A, et al. Identification of Atg5-dependent transcriptional changes and increases in mitochondrial mass in Atg5-deficient T lymphocytes. Autophagy $2009 ; 5: 625$-35.

15. Deretic V, Saitoh T, Akira S. Autophagy in infection, inflammation and immunity. Nat Rev Immunol $2013 ; 13: 722-37$.

16. Ma Y, Galluzzi L, Zitvogel L, Kroemer G. Autophagy and cellular immune responses. Immunity 2013 ; $39: 211-27$.

17. Gros F, Muller S. Pharmacological regulators of autophagy and their link with modulators of lupus disease. Brit. J. Pharmacol. 2014 ; $171: 4337-59$.

18. Arnold J, Murera D, Arbogast F, et al. Autophagy is dispensable for B cell development but essential for humoral autoimmune responses. Cell Death Differ $2016 ; 23: 853-64$

19. Harley JB, Alarcon-Riquelme ME, Criswell LA, et al. Genome-wide association scan in women with systemic lupus erythematosus identifies susceptibility variants in ITGAM, PXK, KIAA1542 and other loci. Nat Genet $2008 ; 40: 204-10$.

20. Han JW, Zheng HF, Cui Y, et al. Genome-wide association study in a Chinese Han population identifies nine new susceptibility loci for systemic lupus erythematosus. Nat Genet $2009 ; 41$ : 1234-7.

21. Lessard CJ, Sajuthi S, Zhao J, et al. Identification of a Systemic lupus erythematosus risk locus spanning ATG16L2, FCHSD2, and P2RY2 in Koreans. Arthritis Rheum 2016 ; 68 : 1197-209.

22. Gros F, Arnold J, Page N, et al. Macroautophagy is deregulated in murine and human lupus $T$ lymphocytes. Autophagy $2012 ; 8: 1113-23$

23. Alessandri C, Barbati C, Vacirca D, et al. T lymphocytes from patients with systemic lupus erythematosus are resistant to induction of autophagy. FASEB J $2012 ; 26: 4722-32$.

24. Clarke AJ, Ellinghaus $U$, Cortini $A$, et al. Autophagy is activated in systemic lupus erythematosus and required for plasmablast development. Ann Rheum Dis $2015 ; 74$ : 912-20.

25. Mizushima N, Yoshimori T, Levine B. Methods in mammalian autophagy research. Cell $2010 ; 140$ : 313-26.

26. Klionsky DJ et al. Guidelines for the use and interpretation of assays for monitoring autophagy. Autophagy 2016; 12 : 1-222.

27. Cuervo AM, Wong $\varepsilon$. Chaperone-mediated autophagy : roles in disease and aging. Cell Res 2014 ; 24: 92-104.

28. Macri C, Wang F, Tasset I, et al. Modulation of deregulated chaperone-mediated autophagy by a phosphopeptide. Autophagy $2015 ; 11: 472-86$

29. Nedjic J, Aichinger M, Emmerich J, et al. Autophagy in thymic epithelium shapes the T-cell repertoire and is essential for tolerance. Nature 2008 ; 455 : 396-400.

30. Dengjel J, Schoor 0, Fischer R, et al. Autophagy promotes MHC class II presentation of peptides from intracellular source proteins. Proc Natl Acad Sci USA 2005 ; 102 : 7922-7.

31. Münz C. Autophagy proteins in antigen processing for presentation on MHC molecules. Immunol Rev $2016 ; 272: 17-27$

32. Deffit SN, Blum JS. A central role for $\mathrm{HSC70}$ in regulating antigen trafficking and MHC class II presentation. Mol Immunol $2015 ; 68: 85-8$.

33. Roche PA, Furuta K. The ins and outs of $M H C$ class II-mediated antigen processing and presentation. Nat Rev Immunol 2015 ; $15: 203-16$.

34. Shibutani ST, Saitoh T, Nowag H, et al. Autophagy and autophagy-related proteins in the immune system. Nat Immunol $2015 ; 16: 1014-24$

35. Blander JM, Medzhitov R. Regulation of phagosome maturation by signals from toll-like receptors. Science $2004 ; 304$ : 1014-8
36. Delamarre L, Couture R, Mellman I, Trombetta ES. Enhancing immunogenicity by limiting susceptibility to lysosomal proteolysis.J Exp Med 2006 ; 203 : 2049-55

37. Moffat JM, Mintern JD, Villadangos JA. Control of MHC II antigen presentation by ubiquitination. Curr Opin Immunol $2013 ; 25$ : 109-14.

38. Cho KJ, Roche PA. Regulation of MHC Class II-Peptide Complex Expression by Ubiquitination. Front Immunol $2013 ; 4: 369$.

39. Martinez J, Almendinger J, Oberst A, et al. Microtubule-associated protein 1 light chain 3 alpha (LC3)-associated phagocytosis is required for the efficient clearance of dead cells. Proc Natl Acad Sci USA 2011; 108 : 17396 401

40. Romao S, Gasser N, Becker AC, et al. Autophagy proteins stabilize pathogencontaining phagosomes for prolonged MHC II antigen processing. J Cell Biol $2013 ; 203: 757-66$.

41. Martinez J, Cunha LD, Park S, et al. Noncanonical autophagy inhibits the autoinflammatory, lupus-like response to dying cells. Nature 2016 ; 533 $115-9$

42. Majai G, Kiss $\varepsilon$, Tarr T, et al. Decreased apopto-phagocytic gene expression in the macrophages of systemic lupus erythematosus patients. Lupus 2014 $23: 133-45$.

43. Page N, Gros F, Schall N, et al. HSC70 blockade by the therapeutic peptide $\mathrm{P} 140$ affects autophagic processes and endogenous $\mathrm{MHCll}$ presentation in murine lupus. Ann Rheum Dis $2011 ; 70: 837-43$.

44. Renna M, Jimenez-Sanchez M, Sarkar S, Rubinsztein DC. Chemical inducers of autophagy that enhance the clearance of mutant proteins in neurodegenerative diseases. J Biol Chem 2010 ; 285 : 11061-7.

45. Fleming A, Noda T, Yoshimori T, Rubinsztein DC. Chemical modulators of autophagy as biological probes and potential therapeutics. Nat Chem Biol $2011 ; 7: 9-17$.

46. Levine B, Packer M, Codogno P. Development of autophagy inducers in clinical medicine. J Clin Invest 2015 ; 125 : 14-24.

47. Rubinsztein DC, Bento CF, Deretic V. Therapeutic targeting of autophagy in neurodegenerative and infectious diseases. J Exp Med $2015 ; 212$ : 979-90.

48. Monneaux F, Lozano JM, Patarroyo ME, et al. T cell recognition and therapeutic effects of a phosphorylated synthetic peptide of the 70K snRNP protein administered in MRL/Ipr lupus mice. Eur J Immunol $2003 ; 33$ : 287-96.

49. Dieker J, Cisterna B, Monneaux F, et al. Apoptosis changes the phosphorylation status and subcellular localization of the spliceosomal autoantigen U1-70K. Cell Death Diff 2008 ; $15: 793-804$.

50. Page N, Schall N, Strub JM, et al. The spliceosomal phosphopeptide P140 controls the lupus disease by interacting with the HSC70 protein and via a mechanism mediated by $\gamma \delta$ T cells. PloS One $2009 ; 4$ : e 5273.

51. Bozzacco L, Yu H, Zebroski HA, et al. Mass spectrometry analysis and quantitation of peptides presented on the MHC II molecules of mouse spleen dendritic cells. J Proteome Res 2011 ; 10 : 5016-30.

52. Dengjel J, Høyer-Hansen M, Nielsen MO, et al. Identification of autophagosome-associated proteins and regulators by quantitative proteomic analysis and genetic screens. Mol Cell Proteomics 2012 ; 11 : M111.014035.

53. Mathew R, Khor S, Hackett SR, et al. Functional role of autophagy-mediated proteome remodeling in cell survival signaling and innate immunity. Mol Cell $2014 ; 55: 916-30$.

54. Schall N, Wang F, Dali H et al. Chaperone-mediated autophagy downregulation normalizes the immune responses in lupus. 2017 (en révision).

55. Zimmer R, Scherbarth HR, Rillo OL, et al. Lupuzor/P140 peptide in patients with systemic lupus erythematosus : a randomised, double-blind, placebocontrolled phase Ilb clinical trial. Ann Rheum Dis $2013 ; 72$ : 1830-5

56. Sarkar S, Davies JE, Huang Z, et al. Trehalose, a novel mTOR-independent autophagy enhancer, accelerates the clearance of mutant huntingtin and alpha-synuclein. J Biol Chem 2007 ; 282 : 5641-52.

57. Mardones P, Rubinsztein DC, Hetz C. Mystery solved : Trehalose kickstarts autophagy by blocking glucose transport. Sci Signal $2016 ; 9$ : fs2.

58. Muller $\mathrm{S}$, Wallace DJ. The importance of implementing proper selection of excipients in lupus clinical trials. Lupus $2014 ; 23: 609-14$.

59. Schall N, Muller $\mathrm{S}$. Resetting the autoreactive immune system with a therapeutic peptide in lupus. Lupus $2015 ; 24: 412-8$.

60. Dragin N, Le Panse R, Berrih-Aknin S. Predisposition aux pathologies autoimmunes : les hommes ne manquent pas d'Aire. Med Sci (Paris) 2017 ; 33 : $169-75$.

\section{TIRÉS À PART}

S. Muller 